\title{
De la documentation aux Humanités numériques : mettre en ligne un fonds documentaire $\mathrm{du}$ $\mathrm{XX}^{\mathrm{e}}$ siècle
}

From documentation to digital humanities: putting a twentieth-century archive online

\section{Arnaud Millereux et Vincent Rousseau}

\section{(2) OpenEdition}

\section{Journals}

Édition électronique

URL : https://journals.openedition.org/cel/19704

DOI : $10.4000 /$ cel. 19704

ISSN : 2262-208X

Éditeur

École du Louvre

\section{Référence électronique}

Arnaud Millereux et Vincent Rousseau, « De la documentation aux Humanités numériques : mettre en ligne un fonds documentaire du xxe siècle ", Les Cahiers de l'École du Louvre [En ligne], 17 | 2021, mis en ligne le 18 novembre 2021, consulté le 13 décembre 2021. URL : http://journals.openedition.org/cel/ 19704 ; DOI : https://doi.org/10.4000/cel.19704

Ce document a été généré automatiquement le 13 décembre 2021.

\section{cc) (†) $९$}

Les Cahiers de l'École du Louvre sont mis à disposition selon les termes de la licence Creative Commons Attribution - Pas d'Utilisation Commerciale - Pas de Modification 4.0 International. 


\title{
De la documentation aux Humanités numériques : mettre en ligne un fonds documentaire $\mathrm{du} \mathrm{Xx}^{\mathrm{e}}$ siècle
}

\author{
From documentation to digital humanities: putting a twentieth-century archive \\ online
}

Arnaud Millereux et Vincent Rousseau

1 Depuis le début du $\mathrm{xxI}^{\mathrm{e}}$ siècle, le développement des outils numériques a bouleversé notre vie quotidienne et plus particulièrement notre accès aux œuvres et aux savoirs. Pourtant, les professionnels des sciences de l'information, bibliothécaires, documentalistes, informaticiens, savent que l'immédiateté et la gratuité de cet accès ne sont qu'une illusion. Les publications en ligne, lorsqu'elles sont rigoureuses, ne demandent pas moins de travail préparatoire que les publications imprimées. Pour devenir accessible sous la forme d'une base de données sur le web [Corpus SATHMA (Sculpture de l'Antiquité Tardive et du Haut Moyen Âge)], le fonds documentaire du projet de Recueil général des monuments sculptés en France pendant le haut Moyen Âge nécessite une patiente et minutieuse étude. Ce fonds résulte de plus de vingt ans de recherches menées principalement par Élisabeth Chatel, Denise Fossard et May Vieillard-Troïekouroff, parfois aidées par d'autres chercheurs, notamment pour la publication des quatre volumes du Recueil général.... Non seulement ce fonds livre une très importante quantité d'informations sur des œuvres parfois négligées, vieilles de plus de mille ans, mais en plus, il témoigne des méthodes de travail des chercheuses du groupe d'études préromanes voulu par Jean Hubert et André Grabar. Après l'étude et la sélection des documents, il faut s'interroger sur les pratiques à adopter pour assurer leur diffusion en ligne et leur pérennité. 


\section{La nécessité de classer ce fonds hétéroclite pour le cerner}

En 2004, le département des sculptures du musée du Louvre a reçu en don la documentation de May Vieillard-Troïekouroff jusqu'alors conservée par sa famille. On suppose qu'elle contient la documentation d'autres chercheurs, parmi lesquels Denise Fourmont et Denise Fossard. En 2009, ce fonds est complété par la documentation d'Élisabeth Chatel. L'ensemble vaste, hétéroclite, difficile à appréhender, a nécessité plusieurs phases de classement par des stagiaires ${ }^{1}$ successifs au département des sculptures. Les fonds Chatel et Vieillard-Troïekouroff occupent environ cent-cinquante boîtes d'archives (soit dix mètres linéaires) classées par départements puis, pour chaque département, par communes et par institutions, conservant ainsi le parti pris par les rédacteurs du Recueil général...

3 À ces boîtes d'archives s'ajoute le fichier préroman: vingt-neuf boîtes de formats différents en métal ou en bois contenant des fiches bristol de vingt centimètres de haut et douze centimètres et demi de large classées par départements puis communes (fig. 1). Au recto de chacune de ces fiches, est collée la photo d'une œuvre accompagnée d'une description. Au verso, un cachet "fichier préroman» accueille un numéro d'identification. Certaines de ces fiches ont été extraites du fichier et intégrées à la documentation du Recueil général..., peut-être comme un travail préparatoire. Cependant, le fichier demeure anonyme et non daté, bien que la date de prise de vue indiquée sur certaines fiches nous livre un terminus post quem. On ignore quels sont précisément les liens entre ces deux ensembles.

Fig. 1

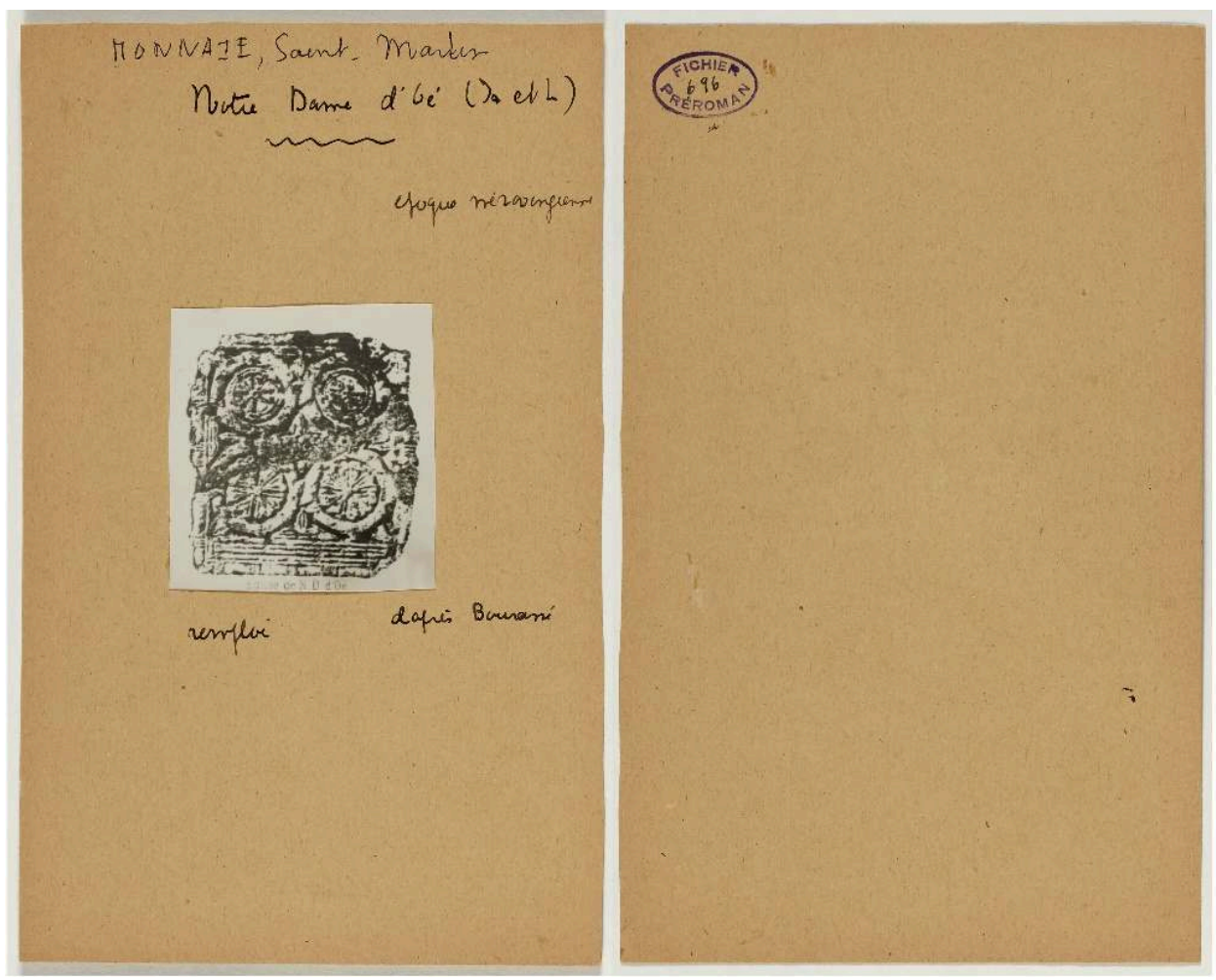

Fiche préroman n 696, dalle carolingienne de Monnaie (Indre-et-Loire). 
Enfin, le fonds est complété par un ensemble de quatre-vingt-trois boîtes en carton contenant chacune une centaine de tirages photographiques. La majorité de ceux-ci sont des doubles d'images déjà présentes dans les boîtes d'archives mentionnées cidessus. Cependant, un sondage commencé en 2021 montre que certains d'entre eux ne se retrouvent pas ailleurs. Ce fonds photographique n'est donc pas à négliger.

\section{La méthode de travail des chercheuses des années 1970 et 1980}

5 La durée particulièrement longue de la recherche, l'importance de l'espace couvert et le nombre conséquent de partenaires expliquent la variété du fonds. L'esprit des chercheuses pouvant être sollicitée à n'importe quel moment, les notes sont prises sur des supports d'une surprenante variété. Aux feuilles de différents grammages et de différentes teintes s'ajoutent des couvertures de bloc-notes, des pages arrachées à des agendas ou des éphémérides, des enveloppes ou, de façon surprenante, des documents administratifs caducs, par exemple des fiches de recensement des hommes de seize à soixante ans datant de la fin de la Première Guerre mondiale ${ }^{2}$. Dans un souci d'économie caractéristique des personnes ayant connu la précarité des années de guerre et d'après-guerre, ces chercheuses recyclaient le papier bien avant l'émergence des mouvements écologistes. En plus des informations sur la sculpture du haut Moyen Âge, ce fonds contient donc des témoignages sur les usages d'une époque qui, sans être lointaine, est déjà fort différente de la nôtre.

6 L'hétérogénéité pittoresque du fonds ne doit pas, cependant, occulter la méthode rigoureuse du groupe de nos prédécesseures. Une première recherche est organisée à distance: d'une part des recherches bibliographiques et, d'autre part, une prise de contact par correspondance avec les sociétés savantes ou érudits locaux. Dans un deuxième temps, les chercheuses se rendent sur place pour prendre des photos de chaque objet dont on conserve des tirages de treize sur dix-huit centimètres et remplir ce que nous appelons désormais une «fiche du recueil» (fig. 2) : fiche cartonnée de vingt-sept centimètres de haut et vingt-et-un centimètres de large où les catégories d'informations à renseigner (commune, objet, dimensions, bibliographie, etc.) sont préimprimées. Pour les chercheurs actuels, posséder la photo et la « fiche-recueil » d'une œuvre est particulièrement satisfaisant. 
Fig. 2

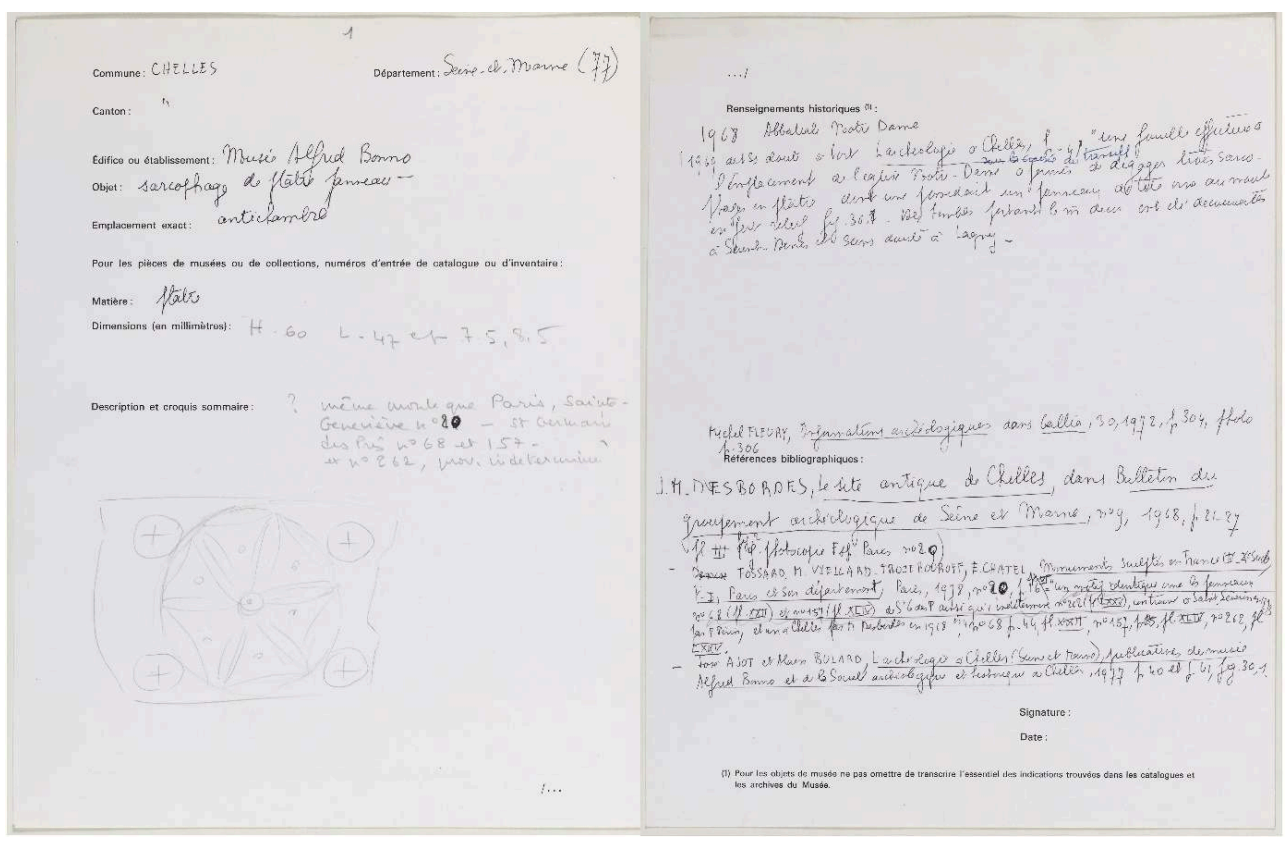

«Fiche du recueil », panneau fragmentaire de tête de sarcophage conservé au musée Alfred-Bonno à Chelles (Seine-et-Marne).

7 Ces fiches ne sont pas toujours remplies scrupuleusement, sans doute, entre autres, par manque de temps. Cette lacune d'information peut cependant être comblée par d'autres documents, comme les «fiches préromanes» ou des ressources bibliographiques.

On déplore cependant que certaines œuvres restent peu documentées. Ainsi, trois sarcophages présents à Noyant-Villages (Maine-et-Loire) ne sont connus que par un courrier du 18 avril 1962. Ils n'ont sans doute jamais fait l'objet d'une enquête de terrain. Pour nous assurer de leur présence, nous devrons mettre nos pas dans ceux de l'équipe du "groupe d'études préromanes " et interroger à notre tour autorités et érudits locaux. Quoi qu'il en soit, l'hypothétique disparition de ces sarcophages n'exclut pas une intégration dans la base de données. En effet, les monuments disparus doivent aussi être publiés. Telle était déjà la démarche de Jacques Sirat, May VieillardTroïekouroff et Élisabeth Chatel. Ainsi dans le tome III du Recueil général des monuments sculptés en France pendant le haut Moyen Âge, une notice est consacrée à un ensemble de quinze stèles découvertes à Ableiges, dans l'actuel Val-d'Oise, connues par une photo de 1887. Cependant, seules quatre d'entre elles étaient conservées avec certitude au musée archéologique du Vexin (actuel Musée archéologique du Val-d'Oise) lors de la publication du volume en 1984. Les autres stèles de cet ensemble ont peut-être été victimes des bombardements de la Seconde Guerre mondiale.

\section{Sélectionner les documents pour la numérisation}

Le cadre géographique et chronologique de la recherche est cependant plus restreint que celui du groupe de travail des années 1970. En effet, on trouve dans le fonds des notes sur des œuvres conservées et créées hors de France ou appartenant aux périodes romane ou gothique, comme les notes sur l'église de Tavant, en Indre-et-Loire, bel 
exemple de peintures et de portail romans. Ces éléments ne font pas partie de notre sujet d'étude. De même, par intérêt pour le contexte de production, de découverte ou de conservation des œuvres, le fonds contient des notes sur le mobilier funéraire, les fouilles récentes ou anciennes, l'histoire et l'architecture de sites remontant à la période qui nous concerne, comme l'église Saint-Pierre de Parçay-Meslay datée du $\mathrm{X}^{\mathrm{e}}$ siècle, mais dont on ne conserve pas de décor sculpté. Là encore, il s'agit d'éléments qui ne sont pas voués à intégrer la base de données SATHMA.

10 La question, récurrente chez les chercheurs, des limites du champ de la sculpture se pose à nouveau ici. Ainsi, lors de l'étude des réserves de Guiry-en-Vexin, une chercheuse des années 1970 s'est interrogée sur un fragment de sarcophage en plâtre, laissant cette note dans les archives : «Est-il vraiment décoré ? ». Autrement dit, les quelques traces apparentes sur la surface sont-elles un accident de coulée, une altération ou un décor intentionnel ? Sauf erreur de notre part, ce fragment est absent du tome III du Recueil général... qui traite des œuvres conservées dans le Val-d'Oise. De même, nous nous sommes interrogés sur les stries présentes sur certains sarcophages, comme celui aujourd'hui conservé dans la nef du sanctuaire carolingien de Cravant-lesCoteaux, en Indre-et-Loire. Bien qu'il soit impossible de connaître les intentions du tailleur-sculpteur et du commanditaire, nous considérons qu'il s'agit d'un décor volontaire et non pas de traces d'outils.

11 Par ailleurs, afin d'éviter les redondances, un document n'est numérisé que s'il contient des informations absentes d'un autre document. Par exemple, le sarcophage AP $112 \mathrm{du}$ musée Carnavalet a été répertorié trois fois dans le fichier préroman: une fiche présente une photo du panneau de pied du sarcophage, une autre fiche une photo du panneau de tête et une troisième, une photo de chacun de ces éléments, les informations écrites d'une fiche à l'autre étant peu ou prou semblables. Dans ce cas, on ne garde que la fiche qui fait la synthèse des deux premières.

La liste des documents à numériser est dressée grâce à un tableur où chaque colonne correspond à une caractéristique normalisée contribuant à l'identification de l'œuvre (localisation, provenance, dénomination, etc.) et chaque ligne à un fichier numérique et non pas au document papier. Par exemple, nous conservons trois documents au sujet d'Esves-le-Moutier, commune d'Indre-et-Loire : deux tirages photographiques et une fiche du recueil. Or, nous souhaitons obtenir une numérisation du recto et du verso de chacun de ces documents. Pour trois documents, on inscrit donc six lignes dans notre tableur. Cette démarche permet de connaitre le nombre de fichiers numériques créés en fin de traitement, d'estimer les frais de numérisation, facilite le dialogue avec la société Azentis, chargée de cette tâche et anticipe le versement des documents dans la base de données en ligne SATHMA.

13 Cette somme d'archives constitue l'assise d'un corpus numérique destiné à être actualisé et enrichi par les travaux et découvertes réalisés depuis les années 1980 et à être mis à la disposition du public grâce à la base de données SATHMA. Un certain nombre de principes ont présidé à sa conception : l'organisation des informations, la préparation de différents formats de données en vue d'une intégration ergonomique dans le système de publication dédié, les outils utilisés pour faciliter l'interopérabilité avec les bases de données existantes et créer un système collaboratif en ligne. 


\section{Méthodologie de la création de fiches}

La création d'une fiche (première méthode, le standard, cas $\mathrm{n}^{\circ} 1$ ) peut s'effectuer directement en ligne en utilisant les formulaires «Sculpture» ou «Sculpture (ensemble) » pour la saisie des données en fonction de la nature de la sculpture décrite et de la granularité de description que l'on souhaite appliquer. Il est important de signaler que leur contenu est basé sur la fiche métier appelée « fiche papier » qui a été validée au sein du groupe de travail en fonction des expériences et retours de chacun qui ont pu apparaitre au cours du temps, des sessions de travail ou des retours des collaborateurs en début de projet. Cette fiche permet aux chercheurs d'organiser leurs données dès le début en suivant une trame commune à tous les objets inventoriés. Une série de critères regroupés par classe a été définie. On retrouve le lieu de conservation et de découverte (lieu décrit précisément, emplacement exact, numéro d'inventaire, statut de l'objet), l'historique ou le remploi de l'œuvre, ses caractéristiques physiques (matériau, dimensions, état de conservation, ensemble ou édifice de rattachement), une description précise (morphologie, technologie, iconographie/décor, présence éventuelle d'inscriptions), des informations sur sa datation ou les discussions autour de ce sujet, les documents associés (photos, notes) avec leurs données Exif ${ }^{3}$ principales, le crédit ou une légende, des sources historiques et d'identification (bibliographie, sitographie, littérature grise), ainsi que des informations sur l'auteur, le statut et la qualité de la fiche. Comme on peut le constater, ce style de fiche s'inspire largement de celui utilisé pour décrire les édifices de la base CARE afin d'assurer une compatibilité par l'uniformisation des données entre les bases déjà existantes. C'est un premier pas vers l'informatisation, et pas des moindres, déjà franchi.

Nous utilisons la fiche «Sculpture (ensemble) » pour associer au sein d'une même entité plusieurs fiches « Sculpture ». Dans le cas, par exemple, de chapiteaux détaillés par face, nous obtenons ainsi quatre fiches «Sculpture" et une fiche «Sculpture (ensemble) ». Des formulaires de saisie viennent surpasser l'éditeur par défaut présent en standard dans le wiki. Ils disposent d'assistants à la saisie reliés à des listes d'autorité afin de préserver au mieux l'homogénéité des données renseignées et affichées. Aucune connaissance technique n'est requise au préalable puisqu'aucun recours à du code n'est nécessaire : la saisie et la mise en page a posteriori repose sur la stratégie en place et est donc gérée par le système; or rien n'empêche l'utilisateur d'utiliser la syntaxe wikitext dans les champs en texte libre, s'il la maîtrise. Les formulaires garantissent une représentation globale consistante et homogène des données. Toute personne disposant d'un compte personnel, créé sur demande, peut ajouter ou modifier des fiches. Dans ce mode d'utilisation, chaque image ou document associé doit être chargé individuellement sur le serveur et relié manuellement pour créer la galerie d'images par le biais des sous-formulaires de création de galeries d'images. Dès lors, des problèmes d'ergonomie sont rapidement mis en avant lorsque de nombreuses fiches doivent être crées et qu'un nombre important d'objets numériques sont à associer.

17 Afin d'améliorer ce point, trois méthodes d'importation sont mises à disposition de l'opérateur dans le but d'aider à «préparer le terrain » avant intégration. À l'issue de l'inventaire, on obtient un ensemble de fichiers de type «texte» et/ou «tableur» 
contenant les informations permettant de créer des fiches par lot ou en batch dans la base de données. Suivant les éléments dont dispose le chercheur, un cas d'utilisation est associé et ceci permet d'aider à déterminer lequel il est préférable d'appliquer. En suivant l'un des procédés, il devient alors concevable d'automatiser un maximum de tâches en fonction de la situation dans laquelle l'utilisateur se trouve. Le premier cas (cas $n^{\circ} 2$ ) est plutôt orienté vers les données. Les chercheurs disposant déjà des informations nécessaires peuvent organiser les données, si cela n'est pas déjà fait, en s'inspirant ou en utilisant des modèles de fiches métiers au format DOC, DOCX, ODT ou PDF afin qu'ils puissent importer leurs données dans la base (fig. 3).

Fig. 3

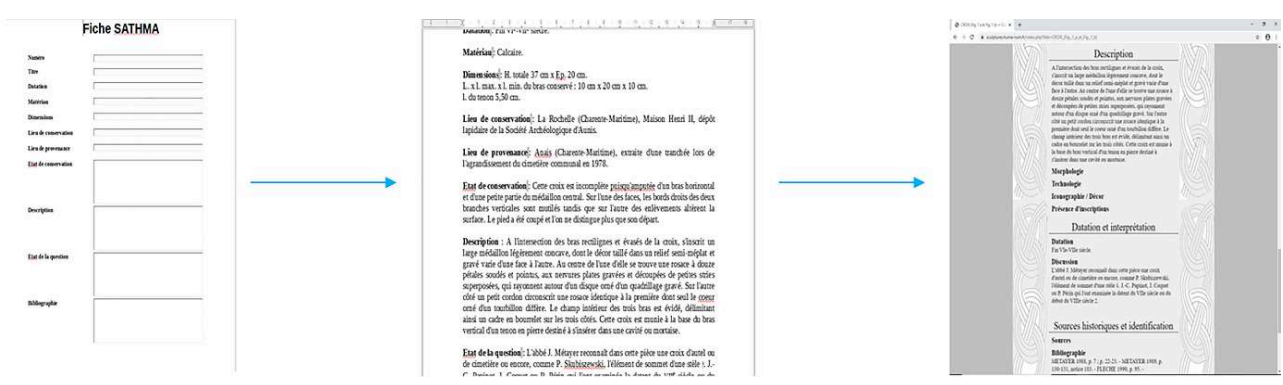

Création d'une fiche complète à partir d'un fichier basé sur un modèle de fiche (traitement de texte).

La première méthode permet de créer aisément des fiches complètes, sans se préoccuper des images associées et est la mieux adaptée pour le mappage (l'association) entre les données de la fiche et la base de données. On conserve une bonne présentation à l'affichage et une bonne ergonomie à la saisie pour le chercheur. La seconde situation ( $\left.\operatorname{cas}^{\circ} 3\right)$ facilite quant à elle l'association des images aux fiches. Dans ce mode de traitement, la préparation sous forme de tableau intervient lorsqu'on souhaite incorporer des images lors de la création des fiches. Les données sont organisées de telle sorte que l'on puisse créer une fiche avec le minimum d'informations. Hormis les données de situation géographique, les informations collectées sont principalement des données techniques de description de l'image, de datation, de matériaux, etc. Outre le fait que le tableau permet de recenser également d'une certaine manière l'ensemble des objets sculptés eux-mêmes regroupés par régions et communes, le tableau liste l'ensemble des images associées à ces objets. Chaque ligne du tableau correspond à un fichier physique issu de la numérisation et représente une page d'un document pour un objet précis en un lieu donné. Dans la base, on obtient une nouvelle fiche par élément "objet»; on aura donc souvent plusieurs documents associés par fiche. Une fiche peut alors rassembler plusieurs lignes $\mathrm{du}$ tableau. Autrement dit, plusieurs lignes sont souvent utiles pour renseigner la fiche de l'objet considéré. Au cours du parcours du tableau, lorsque l'algorithme repère un nouvel objet, une fiche est créée dans la base. Une fiche est créée pour chaque sculpture ou élément sculpté soumis. Dans ce mode, toutes les images de ce même objet sont inscrites et reliées automatiquement à sa fiche. On dispose en outre d'une indication pour mettre au premier plan l'image représentative de l'article considéré et du statut privé ou public de l'image considérée. La fiche comporte un ou plusieurs documents associés, simple ou double face. Les images sont alors associées automatiquement au moment de l'importation du fichier dans le système. Le quatrième cas est une association des deux cas présentés précédemment (cas $n^{\circ} 2$ et cas $n^{\circ} 3$ ) et est utile 
lorsqu'on dispose des fiches de description complètes et d'un tableau listant l'ensemble des documents associés. C'est la forme la plus complète de création automatisée d'une fiche descriptive avec ses ressources associées.

Plusieurs avantages résident dans l'utilisation de ces méthodes. Tout d'abord, l'inventaire étant une étape obligée pour ordonner le fonds, une grande partie du travail déjà faite peut être réutilisée sans recourir à une nouvelle saisie. Cette saisie se déroule en mode déconnecté dans un premier temps pour la partie «Préparation des données ", puis en mode connecté pour l'intégration à la base en ligne et l'association automatique des images aux fiches créées en suivant le référentiel établi lors de l'inventaire par les archivistes, les documentalistes, etc.

Les différents cas exposés permettent une répétition de l'information en conservant une version originale exploitable par le chercheur, tout en facilitant l'intégration dans la base de données. Le schéma ci-dessous (fig. 4) résume les différentes méthodes pour créer des fiches.

Fig. 4

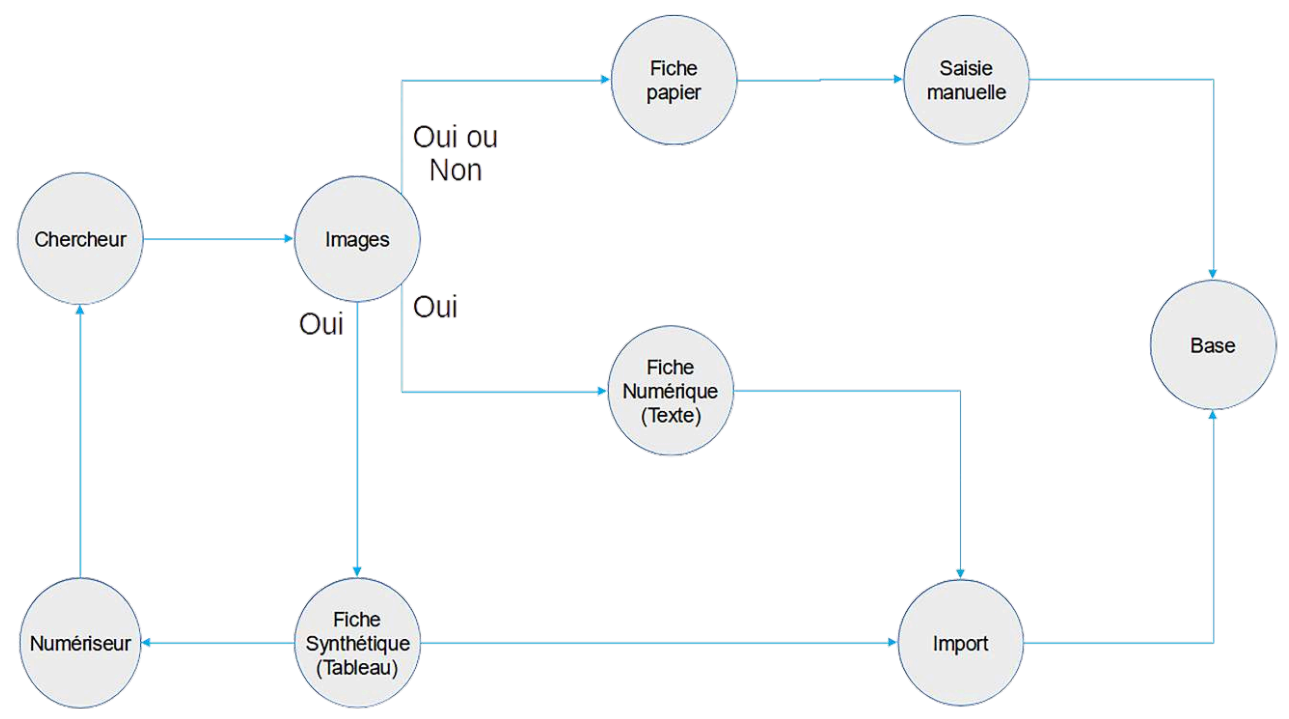

Les différents modes de création d'une fiche.

\section{Mise en ligne des données}

La mise à disposition en ligne des données et des images est assurée par l'outil de gestion de contenu open source MediaWiki ${ }^{4}$, développé avec le langage PHP ${ }^{5}$, outil de type wiki largement utilisé à travers le monde et qui est à la base de WikiPedia ${ }^{6}$, entre autres. Les données sont stockées dans une base $\mathrm{MySQL}^{7}$. L'ensemble est hébergé dans le cadre de ce projet par Huma-Num ${ }^{8}$. Le choix de cet outil est un choix historique. Parmi ses avantages, on peut citer sa robustesse face aux attaques grâce à ses mises à jour régulières, ses nombreuses extensions, ses possibilités d'extension accessibles, ses 
interfaces de programmation API (Application Programming Interface), les liens possibles entre les bases du même type. Pour l'ajout de données, cet outil est exclusivement utilisé en mode d'accès privé.

\section{Gestion des modifications}

À ce jour, les modifications ne sont pas possibles sur la version en production en passant par les fichiers texte ou tableur d'origine. Elle doit s'effectuer directement en ligne par le biais des formulaires de saisie ou le wikitext. L'exportation des données est en revanche intégrée pour disposer d'une sauvegarde.

La gestion des données s'appuie sur les techniques du web sémantique. Elle est assurée en partie par l'extension Semantic Media Wiki', celle des formulaires de saisie par l'extension Page Forms ${ }^{10}$. D'autres fonctionnalités exposées ou en cours de développement utilisent le langage Java ${ }^{11}$ ainsi que la librairie Apache Jena ${ }^{12}$ pour des traitements particuliers sur les informations stockées et les outils recherche, également en vue des futures autres collaborations de façon harmonisée et uniformisée. Les données métiers, dont une partie est commune et partagée avec celles des bases CARE, sont structurées sous forme d'onthologies réalisées à l'aide du logiciel Protégé ${ }^{13}$, luimême développé en Java. Le langage SPARQL ${ }^{14}$ est utilisé pour effectuer les requêtes sur les données enregistrées sous formes de triplets "sujet-prédicat-objet » au sein du triple store (espace où sont stockés physiquement les triplets).

Il est prévu par ailleurs de faciliter l'interopérabilité entre la base de données SATHMA et les bases de données du domaine, en particulier la base des sanctuaires ${ }^{15}$, celle des monastères ${ }^{16}$, ou celle des collégiales ${ }^{17}$, pour laquelle des essais de faisabilité ont déjà été réalisés en amont de façon autonome, sans oublier les bases de données multilingues des corpus CARE, ainsi un cinquième cas d'utilisation sera créé : consulter (et/ou importer) des données par l'intermédiaire d'une interface de programmation (API) fédérant l'ensemble de ces services. Il faut préciser que le lien entre les fiches de la base SATHMA (sculptures) et les fiches de la base CARE (édifices) est déjà disponible depuis quelques temps, puisqu'il est possible de sélectionner l'édifice dans lequel une sculpture doit être répertoriée, depuis la fiche "Sculpture ». Gageons que la mise en ligne de la base de données SATHMA participera d'un renouvellement des recherches sur la création sculptée de l'Antiquité tardive et du haut Moyen Âge.

\section{NOTES}

1. Nous en profitons pour remercier l'ensemble des stagiaires du département des sculptures ayant travaillé sur ce projet jusqu'à ce jour : Florence Cazenave et Élisabeth Ruchaud, puis Maude Barme en 2009, Livia Adjaj et Florence Panciatini en 2014, Sarah Héquette et Grégoire Maquin en 2020, Hugo Quévrin et François Bouquet en 2021. 
2. Imprimés en 1917, ces formulaires n'étaient plus en usage à compter du second semestre de 1919. Nous adressons nos remerciements à M. Christophe Pommier, conservateur adjoint au département Artillerie du Musée de l'Armée pour les informations à ce sujet.
3. Exchangeable image
Exchangeable_image_file_format
file format :
https://fr.wikipedia.org/wiki/

4. https://www.mediawiki.org

5. https://www.php.net

6. https://www.wikipedia.org

7. https://www.mysql.com

8. https://www.huma-num.fr

9. https://www.semantic-mediawiki.org

10. https://www.mediawiki.org/wiki/Extension:Page_Forms

11. https://www.java.com

12. https://jena.apache.org

13. https://protege.stanford.edu

14. https://www.w3.org/TR/rdf-sparql-query

15. Inventaire des sanctuaires et lieux de pèlerinage chrétiens en France: https:// sanctuaires.aibl.fr/

16. Corpus des établissements réguliers de la France médiévale: https://cercornum.univ-stetienne.fr/monasteres/

17. Base des collégiales séculières de France (816-1563) : http://vafl-s-applirecherche.unilim.fr/ collegiales/

\section{RÉSUMÉS}

La documentation réunie par les équipes de recherche du Recueil général des monuments sculptés en France pendant le haut Moyen Âge est conservée au département des sculptures du musée du Louvre. Elle fait l'objet d'un projet de base de données. Cette "dématérialisation " nécessite un long et minutieux travail préparatoire. Il faut d'abord avoir une vision d'ensemble du fonds qui, par ailleurs, témoigne des méthodes de travail systématiques antérieures au développement de l'informatique. Il faut ensuite sélectionner et préparer les documents à numériser. Quatre méthodes différentes sont envisageables pour mettre les documents en ligne par l'outil MediaWiki, en espérant pouvoir assurer l'interopérabilité entre la base SATHMA, adossée à CARE, et les autres bases de données patrimoniales.

The documentation compiled by the Recueil général des monuments sculptés en France pendant le Haut Moyen âge research teams is kept in the Sculpture Department of the Musée du Louvre. There are plans to transform it into a database. This "dematerialisation" requires long and meticulous preparatory work. First, it is necessary to have an overall view of the collection that, moreover, bears witness to the systematic working methods that predate the development of information technology. Then the documents to be digitised must be selected and prepared. Four different methods of putting the documents online using the MediaWiki tool may be envisaged, in the hope of ensuring interoperability between the SATHMA database, which is supplemented by CARE and other heritage databases. 


\section{INDEX}

Mots-clés : archives, bases de données, haut Moyen Âge, humanités numériques, méthodologie, numérisation, sculpture, WikiMédia

Keywords : archives, databases, Early Middle Ages, digital humanities, methodology, digitisation, sculpture, MediaWiki

\section{AUTEURS}

\section{ARNAUD MILLEREUX}

Titulaire d'un DESS Bases de Données et Intelligence Artificielle depuis 2003 et Assistant Ingénieur titulaire en tant que Développeur / Intégrateur d'applications au sein de la Maison des Sciences de l'Homme de Dijon depuis 2012, Arnaud Millereux contribue au développement du programme CARE dont l'ANR s'est achevée en 2011 mais reste très investi dans le projet encore aujourd'hui en le faisant évoluer. Depuis, il intervient également au sein du groupe de travail du projet SATHMA pour relier les deux bases et envisager d'autres ramifications. Par ailleurs, il s'intéresse aux nouvelles technologies en effectuant une veille permanente.

Arnaud Millereux obtained his DESS postgraduate degree in databases and artificial intelligence in 2003. He has been working as an application developer and integrator at the Maison des Sciences de l'Homme in Dijon since 2012, has contributed to the CARE programme whose Agence Nationale de Recherche funding came to an end in 2011 but remains very committed to the project and is still developing it. Since then, he has also been involved in the SATHMA project working group to link the two bases and to consider other ramifications. He is also interested in new technologies and constantly monitors them.

\section{VINCENT ROUSSEAU}

Titulaire d'un Master 2 professionnalisant Métiers du patrimoine de l'École du Louvre en 2008, Vincent Rousseau devient chargé d'études documentaires en 2013. Après avoir été documentaliste pendant cinq ans au musée national de la Renaissance à Écouen (Val-d'Oise), il rejoint le département des Sculptures du musée du Louvre en septembre 2018. Il y est chargé de la documentation sur la sculpture médiévale et participe au projet de recherche SATHMA. The holder of a Master 2 in heritage professions from the École du Louvre (2008), Vincent Rousseau became a researcher in 2013. After having worked as an archivist for five years at the Musée National de la Renaissance in Écouen, Val-d'Oise, he joined the Sculpture Department at the Musée du Louvre in September 2018. He is responsible for documenting medieval sculpture there and also takes part in the SATHMA research project. 